\title{
Interactive comment on "Real-time optimization of wind farms using modifier adaptation and machine learning" by Leif Erik Andersson and Lars Imsland
}

\section{Leif Erik Andersson and Lars Imsland}

leif.e.andersson@ntnu.no

Received and published: 14 April 2020

Dear Bart, Thank you for your very detailed review on our paper. I believe including your comments and recommendation will improve the paper considerably. Here I will respond to your four main points. In addition, I answered the questions in your attached pdf. I will attach the pdf to this comment.

Please see below a more detailed response: Point 1: 1 . The article seems heavily focused on the algorithm. A more in-depth discussion on the practicality of the algorithm is missing. Questions you raised are: a. How can the algorithm be applied to wind farm control on real sites? b. What would you consider a training set in real life? c. Do you need to time average measurements? d. How would you deal with time-varying 
inflow conditions? e. Are time delays due to wake propagation an obstacle?

These are extremely interesting and important questions, which were also raised by the other referee. My plan is to include a discussion section in the revised version of the article to highlight some of these points. We performed also a LES study, which will be presented at the TORQUE 2020. Based on my own expectations and the LES study I can say: The training set would be at least the wind velocity, wind direction, and the power outputs of the turbines. Turbulence intensity could be considered as an input. I would recommend doing a sensitivity study to evaluate how much the variance in the turbulence intensity affects the outputs (it should be considerably larger than the affect of the input noise in the wind measurements). The algorithm needs time averaged measurements. Without an appropriate filter the variance in the data will be large, which will degrade the performance of the learning algorithm. In the LES study we used 5-minutes averaging, which was enough. However, we used quasi-static inflow conditions without wind direction changes. Wind direction changes will degrade the performance. In general, input noise can be to some extend counteracted with more data (assuming the input noise is not biased). I am unsure how the algorithm will react on biased data. If the bias is consistent - e.g. 5 degrees of in all measurements - it should not degrade the algorithm. For a real-life application, it would be necessary to first collect data. The wind direction can be included as an input variable. Otherwise, for many small circle sectors a new model would have to be identified, which could be impractical. I expect, it is necessary to differentiate between atmospheric conditions and identify separate models for each of these conditions. A multi-model approach could be used. Time delays are difficult to handle. Steady-state data is wanted so it is necessary to wait until the first downstream turbine is affected by changes in the upwind turbine. It is difficult to evaluate how much time delays will degrade the performance of the algorithm. In simulation we performed it seems most of the energy transfer in the plant can be captured with the upstream and the next two downstream turbines.

Point 2: 2 . The article can be significantly reduced especially the sections about the

Interactive comment 
MA and GP algorithms can be condensed. I see you point and will condense these sections. I will also try to shift the focus from the algorithms towards the application.

WESD

Point 3: 3. The literature survey presented in the introduction seems to focus on general wind farm control. It would be useful to shift to surrogate modelling and adaptation in wind farms. I will try to include these references and refocus the introduction on these topics. The literature on surrogate modeling creating new engineering wake models is quite large. On the other hand, only a few authors considered adaptation of these models for wind farm control. Some considered parameter estimation for their specific wind farm control application. Many used the parameters supplied by the wind farm model (also often estimated from LES data etc.).

Point 4: 4. Shorten the article, clearly state the contribution in the introduction and also start each section with one or two sentences relating the upcoming section to the previous section. In addition, it may be helpful to gather some information in tables. This can be easily included in the revised version of the article. It also is related to point 2.

Again, thank you for all your comments. I will do my best to include them in the revised version.

Please also note the supplement to this comment:

https://www.wind-energ-sci-discuss.net/wes-2020-18/wes-2020-18-AC1-

supplement.pdf

Interactive comment on Wind Energ. Sci. Discuss., https://doi.org/10.5194/wes-2020-18, 2020.

Interactive

comment

Printer-friendly version

Discussion paper 